\title{
Tecnologías digitales, comunidades virtuales y nuevas formas de leer ¿Qué está pasando en el sector editorial juvenil en Argentina? ${ }^{2}$
}

\author{
Digital Technology, Online Communities and New Ways of Reading \\ What's Happening in Argentina's Youth Publishing Field?
}

\author{
PAULA CUESTAS \\ CONICET-CIMeCS/IdIHCS-UNLP \\ VICTORIA SAEZ \\ CONICET-IIGG-UBA-FLACSO \\ Argentina \\ paula.cuestas9o@gmail.com \\ saezvictoria@live.com
}

(Recibido: II-II-2OI9;
aceptado: I5-O5-202O) $^{-0}$

Resumen. El sector editorial orientado al público juvenil es uno de los que más ha crecido en los últimos años en ventas y publicaciones a nivel mundial y también en la Argentina. En paralelo a este fenómeno, los avances tecnológicos modificaron las prácticas de lectura y escritura, sobre todo entre los jóvenes: plataformas dedicadas a la literatura, enormes comunidades virtuales de lectores y nuevos mediadores de lectura en las redes, como los BBB (bloggers, booktubers y bookstagrammers), configuran este mapa novedoso. El sector ha percibido estas nuevas experiencias incorporando estrategias para capitalizarlas. El presente trabajo se propone realizar un acercamiento a esta configuración editorial que emerge en el sector juvenil haciendo énfasis en los nuevos actores y acciones de promoción. Las afirmaciones de este artículo están basadas en un abordaje metodológico de tipo cualitativo, a partir del análisis de registros de campo en eventos como ferias del libro, así como de la realización de entrevistas en profundidad a miembros de la comunidad BBB, referentes del sector editorial juvenil y a jóvenes lectores que consumen tanto libros como BBB.

Palabras clave: jóvenes; lecturas; tecnologías digitales; industria editorial.
Abstract. The youth publishing field is one of the fastest growing in sales and publications in the world, and also in Argentina in the last years. Parallel to this phenomenon, technological advances have modified reading and writing practices, especially among young people: Platforms dedicated to literature, huge virtual communities of readers and new mediators of reading on networks, such as BBB (bloggers, booktubers and bookstagrammers), make up this novel map. The sector has perceived these new experiences by incorporating strategies to capitalize on them. This paper proposes an approach to this editorial configuration that emerges in the youth sector with emphasis on new actors and promotional actions. The statements in this article are based on a qualitative methodological approach, from an analysis of fieldwork at events such as book fairs, as well as in-depth interviews with members of the BBB community, references in the youth publishing sector and young readers who consume both: books and BBB.

Keywords: young people; reading; digital technologies; publishing industry.

\footnotetext{
${ }^{\text {I }}$ Para citar este artículo: Cuestas, P. y Saez, V. (2020). Tecnologías digitales, comunidades virtuales y nuevas formas de leer. ¿Qué está pasando en el sector editorial juvenil en Argentina? Álabe 22 . [www.revistaalabe.com]

DOI: IO.I5645/Alabe2O20.22.9

${ }^{2}$ Agradecemos a Ezequiel Saferstein sus agudos y precisos comentarios a ponencias presentadas por ambas autoras en las II Jornadas de Comunicación y Cultura del IDAES y en las XII Jornadas de Sociología de la UBA, ambas durante el mes de agosto de 2OI9. Aquellos trabajos preliminares, así como los aportes de nuestro comentarista, han servido de insumos para la redacción de este artículo.
} 


\section{Introducción}

Al ingresar a la Feria Internacional del Libro (FIL) de la Ciudad Autónoma de Buenos Aires (CABA), es posible toparse con un paisaje diverso: editoriales con perfiles muy disímiles y propuestas a veces antagónicas pueblan durante el mes de mayo y por tres semanas el gran predio ferial de Av. Santa Fe. En el pabellón más grande de la Sociedad Rural, ni bien se ingresa desde el acceso principal que conecta con los otros edificios, se accede a un llamativo stand que suele desbordar de personas. Se trata de la librería Cúspide (parte del Grupo Clarín a partir del año 2OII) que desde el 2018 tiene una stand especialmente dedicado a su sección juvenil, en un lugar estratégico dentro del predio.

La FIL se mueve al ritmo de los más jóvenes. Si echamos un vistazo al programa de actividades, gran parte del cronograma está dedicado a personas que tienen entre I2 y 30 años. En la edición de 2019 estuvieron presentes numerosos autores internacionales (generalmente norteamericanos) asociados al género juvenil. Las presentaciones de sus libros así como las charlas que oficiaron fueron de las más convocantes a lo largo de las tres semanas que dura el evento. Las actividades propulsadas desde el área juvenil de Fundación El Libro (organizadora de la FIL) incluyeron, además de la presencia de estos autores (propuestas hechas en vínculo con distintas editoriales así como con la embajada de Estados Unidos), encuentros con bloggers, booktubers y bookstagrammers (la comunidad BBB) y charlas sobre temáticas que interpelan a la juventud: el lenguaje inclusivo, el cuerpo en la literatura juvenil, Harry Potter (HP) y el canon creado por J. K. Rowling, debates sobre poesía, entre otras.

Los jóvenes leen. Detrás del mito que sostiene que no lo hacen, se encuentra un imaginario que aún asocia el acto de leer con una figura solitaria y reflexiva propia del paradigma posmoderno, al decir de Papalini (2O2O). En ese cuadro no hay lugar para estas prácticas de carácter colectivo, creativo, que involucran una amplia gama de actores sociales y que no se detienen en el libro sino que, por el contrario, suponen un vínculo activo también con las nuevas tecnologías digitales. En ese imaginario se le atribuye a la escuela el monopolio de los procesos de socialización, en particular el control de los procesos de socialización lectora (Dubet, 2002; Lahire, 2004). En el campo local, el diagnóstico de las ciencias sociales respecto de lo que ha pasado en la escuela a partir de los años '9o señala una doble dimensión de la crisis institucional en la que se encuentra el proyecto escolar: la pérdida de control sobre los procesos de conformación de subjetividades y de su poder de interpelación (Kessler, 2002, 2004). Estos estudios sobre la crisis del sistema educativo generalmente enfatizaban, en particular, las crecientes dificultades de la institución a la hora de "promover la lectura" (Corea y Lewkowicz, 2004).

A partir de ello, en las últimas dos décadas los medios de comunicación construyeron una narrativa sobre la crisis educativa en la que se presenta una imagen de niños y jóvenes como sujetos que “ya no leen”. Desde esta posición, la gran mayoría de las voces que se alzan para acusar una supuesta "pérdida de valores" por la "falta de lectura", reclama por un determinado tipo de literatura, que deja por fuera los productos vinculados 
a la industria cultural masiva (López Corral, 20I4). Tal vez lo más “incómodo" al abordar analiticamente las prácticas lectoras de buena parte de la juventud tenga que ver con el carácter "ilegítimo" de los libros que leen: best sellers, románticos, fantasía, que no se amolda a los estándares tradicionales del "buen libro". De acuerdo con Cuesta y Sawaya (20I6), bajo el diagnóstico que presume que hay textos buenos, que merecen ser leídos, y otros, generalmente vinculados a la gran industria editorial, que no cargan con la misma valoración positiva, se dejan de lado producciones literarias dentro de la currícula canónica.

Desde el campo académico debemos suspender ese juicio estético. Como propone Seman (2015), retomando aportes del pragmatismo francés, suspender las valoraciones normativas (que en este caso se expresan en el canon escolar y anclado en la imaginario de la "buena literatura") puede traer productividad sociológica sobre el fenómeno a analizar. Desde este enfoque nos posicionamos al escribir este trabajo en el que nos proponemos conocer, en primer lugar, el papel que las tecnologías digitales desempeñan en la conformación de prácticas lectoras y de qué modo actualizan los vínculos entre jóvenes y literatura. Luego de esa presentación general, nos abocaremos al análisis de un colectivo en particular: la comunidad BBB, sus prácticas, sus lógicas y su expansión en los últimos años. Finalmente, veremos de qué modo los miembros de esta comunidad se vinculan con la industria editorial y qué continuidades y rupturas hay entre las estrategias que actualmente se emplean en ese sector y las de antaño.

\section{Decisiones metodológicas}

Antes de comenzar a abordar los temas esbozados al cierre de la introducción, resulta preciso hacer una advertencia respecto de las decisiones metodológicas adoptadas para llevar adelante esta investigación. En primer lugar, queremos destacar que al tratarse de un trabajo en co-autoría recuperamos materiales empíricos de ambas autoras, fruto de sus respectivas investigaciones de posgrado. En consecuencia, las afirmaciones de este artículo están basadas tanto en registros de campo de eventos como ferias del libro como en la realización de entrevistas en profundidad a miembros de la comunidad BBB, referentes del sector editorial juvenil y a jóvenes lectores que consumen, generalmente, este tipo de literatura y que siguen cuentas o canales de miembros de la comunidad BBB.

Se trata, por tanto, de un abordaje metodológico que combina más de una técnica de investigación de tipo cualitativo. Por un lado, se trabaja desde una perspectiva etnográfica, la cual entendemos, junto a Peirano (2004), como una apuesta por una puesta en diálogo entre práctica y teoría. En ese sentido, se trata de una técnica de investigación que permite captar, a partir de ese diálogo, los distintos sentidos que los sujetos otorgan a sus prácticas y las diversas experiencias que a partir de ello se habilitan. Concretamente en este artículo, el propósito que se persigue es ver de qué modos estos nuevos actores del campo editorial ingresan y toman un rol en el sector juvenil, con las rupturas y 
continuidades que esto supone. Al mismo tiempo, la posibilidad de realizar entrevistas individuales semi-estructuradas nos permite acceder a conocer situaciones no directamente observables a partir de la observación etnográfica -lo que es destacado por Valles (I997) y Piovani (20I8) como una de las principales ventajas de esta técnica-, como, por ejemplo, el conjunto de decisiones que atañen a la publicación de un libro, las estrategias de marketing y promoción, entre otros tantos. Fue por ello que, a los fines de este trabajo, se realizaron entrevistas a actores claves de este universo. En los casos en que se recuperan, de forma explícita, fragmentos de dichas entrevistas se ha modificado el nombre de nuestras informantes para preservar su anonimato.

Asumimos que la complementariedad de ambas técnicas de investigación nos ha permitido cumplir con los objetivos del presente artículo al recuperar, en sus propios términos, las voces de los propios actores intervinientes en este encuentro entre libros y literatura, dentro del sector juvenil del campo editorial.

\section{Plataformas digitales y su influencia en las prácticas lectoras de los jóvenes}

A la narrativa que sostiene que "los jóvenes no leen", se le suma un discurso pregnante, ampliamente difundido y sedimentado en la sociedad, que sostiene que las prácticas de lectura se encuentran en detrimento como consecuencia del auge de las nuevas tecnologías de la información y la comunicación (TIC) (Gaarder, 2003; Pindado, 2004). Que "los chicos no leen porque están todo el día con el celular" ya es parte del "sentido común" 3 . Lo que estas visiones no consideraron es la complementariedad entre las transformaciones tecnológicas y las múltiples modalidades de acceso a la lectura.

Más allá del arraigo popular de estos imaginarios, desde el campo académico, en la última década, una serie de investigaciones han comenzado a dar cuenta de esta relación mostrando, a su vez, cómo el desarrollo tecnológico ha propiciado nuevas formas de acercamiento a la lectura entre los más jóvenes. En países como España y México, en los que los fenómenos que evidencian la complementariedad entre tecnologías digitales y prácticas lectoras tienen otro alcance y visibilidad ${ }^{4}$, se ha comenzado a consolidar un área de interés académico con la publicación de trabajos empíricos (mayormente de corte cuantitativo) que abordan distintas aristas de este nuevo vínculo entre jóvenes y lectura. En el caso español se destacan los trabajos de Cassany (2OII), Lluch (2OI4), Escandell (2OI6), Rovira Collado, (2OI7), De la Torre-Espinosa (2O2O) así como en el mexicano se reconocen producciones como la de Pacheco Alonso (2OI4) o la compilación dirigida por García Canclini (20I5). En términos generales, estos trabajos enfatizan en la idea de que el entorno digital que nos envuelve hace que cada vez sea más frecuente asociar la mayoría de las actividades relacionadas con la literatura (y por extensión la

\footnotetext{
3 Papalini recupera y profundiza respecto de las aseveraciones del Sentido Común sobre las prácticas lectoras en un interesante artículo publicado en esta misma revista (Papalini, 2OI2b).

${ }^{4}$ Debido, principalmente, a la cantidad de seguidores (sobre lo que profundizaremos en las próximas páginas).
} 
lectura) a internet. Así lo empiezan a ver algunos escritores que ya han comenzado a experimentar desde la red: escriben on line, mantienen contacto con su público merced a su blog o su página web, u optan directamente por la publicación digital eludiendo el soporte en papel (Merino y Polanco, 20I5: 76).

Si bien en Argentina podemos encontrar importantes estudios sobre cibercultura juvenil y su incorporación a la escuela (Ferreiro, 2OII; Dussel, 2OI2, Pini et al., 2OI6), particularmente, los trabajos que estudian la relación particular entre tecnologías digitales y literatura en el universo juvenil son apenas incipientes. Sin embargo, en esta línea, algunas producciones han comenzado a visibilizar las conexiones entre las influencias normativas y técnicas con la emergencia de nuevas disposiciones de ser y habitar el mundo a partir de las experiencias literarias, abonando a la construcción de una nueva figura del lector (Papalini, 2OI2a; 2OI5a). En este terreno, la preocupación por el uso y apropiación de tecnologías digitales ocupa un espacio creciente (Albarello et al., 20I8; Kozac, 20I8; Saez, 20I9).

El desarrollo tecnológico implicó un proceso de recomposición de la cultura a escala mundial que modificó no solo los soportes, sino también a los actores involucrados (Baricco, 20I9). En un contexto de convergencia entre diversos medios de comunicación, soportes y contenidos (Jenkins, 2008) las prácticas de lectura han sido transformadas. Las experiencias literarias juveniles van más allá de la experimentación artística, se erigen como un nuevo tipo de sociabilidad. Y en esa construcción de lazos sociales en redes de escritura literaria y en consumos de libros, se tejen identidades, vínculos, creaciones artísticas experimentales; en definitiva, las tecnologías digitales inauguran un nuevo tipo de literatura y nuevos modos de vincularse con ella. La formación de comunidades virtuales que intercambian gustos literarios y crean textos de manera individual y colectiva; espacios virtuales masivos dedicadas a la lectura, escritura y crítica literarias, como Wattpad, Tumblr, Goodreads, Fandoms y diversas redes sociales; nuevos mediadores de lectura en las redes, intercambios constantes entre lectores y autores, son algunos de los emergentes que constituyen este mapa novedoso.

Esta expansión de las TIC permite, por ejemplo, la promoción de espacios de lectura y la consolidación de foros virtuales de discusión sobre sagas literarias vinculadas a la industria masiva. Experiencias que con los años derivaron en la conformación de numerosos clubs de fans, propiciadores de encuentros colectivos de creación y recreación de distintos fandoms $s^{5}$ a través de la puesta en marcha de múltiples actividades.

La plataforma online, Wattpad, también es un caso emblemático en esta misma dirección. Constituye una comunidad de lectores y escritores que en el mundo tiene más de 60 millones de usuarios y en Argentina casi 2 millones. Esta red implica la interacción

\footnotetext{
5 El término es una contracción de fanatic kingdom (del inglés: reino fan) y refiere a grupos de aficionados, generalmente, en torno a la ciencia ficción y la literatura fantástica que se dan estrategias para recrear estos universos. Lo interesante de su uso es que no se reduce a una categoría propia del mundo académico, sino que es utilizada por los propios fans para enmarcarse como parte de un colectivo (Torti Frugone y Schandor, 20I3).
} 
directa entre escritor y lector en el mismo proceso de la obra, a medida que se suben capítulos de una novela, los lectores pueden comentar y poner en cuestión la obra, volviendo la materialidad del texto de alguna manera maleable a la intervención del lector.

El fenómeno de los fanfictions, predominante en esta red, es asimismo ilustrador. Se trata de relatos de ficción escritos por fans de una obra (ya sea película, novela, programa de televisión, videojuego, anime) en los que se utilizan los personajes, situaciones y ambientes descriptos en la historia original y se crea una nueva trama con nuevos personajes. Es la intertextualidad surgida de los fanáticos.

Cabe aclarar que reconocer la emergencia de este nuevo escenario, no implica desconocer la mercantilización de la red que vuelve a los usuarios consumidores -y objetos de consumo-, imponiendo un sistema de recomendaciones, jerarquía, popularidad (Van Dijck, 2OI3). La fe en el espíritu público, libre, la apertura a diversas voces que ofrecía Internet no evitó que los poderes industriales y económicos se filtraran mediante publicidades, trolls, clickbaits, como un control de la información de la sociedad de consumo. La forma corporativa hegemónica con sus incesantes exigencias de crecimiento pueda llegar a saturar de publicidad los espacios de internet pues los sitios virtuales dominantes aspiran sin descanso a la conquista de nuevos mercados (Sadin, 2018).

Sin embargo, existen puntos de fuga que abren nuevas posibilidades para la creación literaria y su circulación. Por la tanto, se vuelve indispensable poner una mirada sobre estos procesos para analizarlos y reflexionar sobre la posibilidad de nuevas formas de construcción de sentido desde las voces de sus "prosumidores" ${ }^{6}$ en el espacio digital. Nos preguntamos, en consecuencia, de qué modo se reconfiguran las prácticas de lectura a partir de las apropiaciones juveniles de las tecnologías digitales y que líneas de acción habilitan esos vínculos que se entablan entre jóvenes y literatura.

\section{Comunidades en red: el caso de "los BBB"}

En los últimos cinco años, muchos jóvenes que crecieron participando de estos espacios de interacción en red o, al menos, como consumidores de este tipo de literatura, han comenzado a formar parte (y crearon, de hecho) de lo que se conoce como comunidad BBB: bloggers, booktubers y bookstagrammers (Pates, 20I5). Youtube (bajo su lema de "hacernos a nosotros mismos") expandió el mundo de las redes sociales. Allí podemos encontrar personas hablando sobre distintos temas, en distintos registros y apuntando a públicos muy diversos. Entre ellos, muchos jóvenes comenzaron a usar su canal para explayarse sobre los libros que leían generando un fenómeno que dio por denominarse booktubers. Pero los bloggers fueron los pioneros en este camino, cuando a mediados de la primera década del milenio comenzaron a publicar contenidos de manera regular en

\footnotetext{
${ }^{6}$ Este concepto es propuesto por Toffler (I980) para mostrar la interrelación entre estos los procesos de producción y consumo. Fenómeno que se hace visible en el escenario que aquí estamos describiendo, y que arroja claves de indagación para su análisis.
} 
sus blogs, sólo que en este caso hablando específicamente de libros. Finalmente, se utiliza el término bookstagrammers para nombrar a quienes comparten sus lecturas a través de Instagram. En la gran mayoría de los casos, unos y otros se retroalimentan ya que la mayoría de los usuarios hace un uso múltiple y diverso de sus redes sociales.

El cambio profundo en los sistemas de comunicación de la actual "sociedad red" (Castells, 2OOI), en la que la cultura se conforma por contenidos y también por prácticas, ha repercutido de manera directa en los modos en que los jóvenes abordan a la literatura. $\mathrm{El}$ hecho literario se vuelve un hecho social y una excusa para interactuar con los pares. En ese escenario el rol de los BBB es clave: su papel como mediadores entre los libros y otros jóvenes los coloca en un lugar privilegiado para propiciar distintos intercambios en la sociabilidad juvenil. Estos actores, que comenzaron de forma autónoma y lograron una masiva repercusión en jóvenes que siguen sus recomendaciones de lecturas, manejan un código de intimidad con sus seguidores: les piden que sigan sus cuentas, que pongan likes y les responden los comentarios de manera directa. Desde las plataformas virtuales les hablan de libros de papel, muestran sus bibliotecas, las nuevas ediciones y las distintas tapas y las colecciones completas de las sagas.

Sin buscar hacer una generalización que obture los diferentes perfiles que podemos encontrar dentro de esta comunidad, el trabajo de campo con ellos realizado, nos permite afirmar que se trata mayormente de jóvenes (varones, mujeres y personas que se autoperciben con sexualidades disidentes) de entre i8 y 30 años de edad, que habitan en CABA o en el Conurbano bonaerense, que son estudiantes o egresados de universidades o instituciones de nivel terciario (de carácter público y privado), en muchos casos en carreras autopercibidas afines con su práctica lectora (como Licenciatura en Comunicación Social, Licenciatura o Profesorado en Letras y Lenguas modernas, Traductorado de inglés, Edición, entre otras), y que suelen convivir con sus padres (los más jóvenes) o con sus parejas. Pocos de ellos declaran haber necesitado trabajar mientras cursaban sus estudios superiores, la gran mayoría lo hizo, de acuerdo con sus palabras, para "ganar experiencia” (en muchos casos en el mundo editorial). Casi todos dominan el inglés: leen y ven series en ese idioma sin necesitar de un traductor o subtítulos; algunos, inclusive, hacen sus publicaciones en redes sociales en inglés ya que consideran que expande potencialmente su espectro de alcance e influencia. Mayoritariamente, poseen celulares de alta gama (Iphone, los modelos más caros de Samsung, LG o Motorola) con buena conectividad a Internet y buenas cámaras para tomar fotografías. De acuerdo con esta descripción, puede pensarse en ellos como sujetos pertenecientes a los sectores medios de la sociedad argentina (Visacovsky, 20o8).

Las primeras experiencias de booktubers datan del año 2008 en Estados Unidos. En Argentina, el fenómeno comenzó a expandirse más tarde y con otro ritmo y alcance: los booktubers locales más reconocidos tienen un promedio de I5.ooo seguidores en su canal $^{7}$ y se consolidaron o surgieron recién en 20I4, mientras que en España, Colombia

\footnotetext{
${ }^{7}$ Anto Romano tiene 6.500 suscriptores, Natalya I4.400 suscriptores, Matias G.B 22.400 suscriptores, Maca Yanelli tiene $23 \cdot 5$ Oo suscriptores, por citar algunos casos.
} 
o México los referentes en este tema pueden llegar casi al medio millón ${ }^{8}$. Pese a ello, en el plano local, el universo BBB no deja de ser convocante y llamativo. En el último tiempo, muchos booktubers y bloggers han migrado hacia Instagram, proceso que no es exclusivo de los BBB sino común a muchos influencers ${ }^{9}$ que encuentran en esa plataforma el medio por excelencia para expandir el alcance de sus contenidos.

Tal vez el punto de inflexión para estas experiencias en el plano local puede situarse entre 2014 y 2OI ${ }^{\text {IO }}$. En el año 2OI4, vino por primera vez a la FIL de CABA, tal vez el evento editorial más importante de nuestro país, un escritor especialmente convocado para el público juvenil desde la Coordinación de la Movida Juvenil: el norteamericano James Dashner, autor de la popular trilogía The Maze Runner. Al año siguiente, se organizó el primer Encuentro Internacional de Booktubers, congregando a cientos de jóvenes. En 20I9, se celebró su quinta edición. Asimismo, empezaron a desarrollarse las convenciones de bloggers y las charla con bookstagrammers, eventos que cada año son más masivos. Estos encuentros en la feria los coordina y gestiona el área juvenil de Fundación El Libro, en articulación con grandes grupos editoriales como Planeta y Penguin Random House (PRH).

Con los años, muchos de estos jóvenes comenzaron a capitalizar esos espacios como una plataforma laboral. Como señala Melo Moreira (20I8), al emerger como voces autorizadas en las recomendaciones de libros para sus pares, estos nuevos mediadores de lectura comenzaron a profesionalizarse y a ser parte de las estrategias de promoción de las editoriales. Pates (20I5) acompaña este diagnóstico al señalar que la mediación de tecnologías en la escritura y lectura genera que jóvenes de la misma edad se vuelvan referentes claves en recomendaciones literarias, por sobre los adultos.

Muchos de los BBB han sido contratados para trabajar en los grandes grupos editoriales en Argentina. ¿Cuál es el vínculo que establecen estos jóvenes con el mundo editorial de literatura juvenil y masiva? ¿Cómo se re-configura de un modo diferencial ese vínculo cuando se establece una relación laboral entre estos jóvenes y las editoriales bajo la figura emergente de "directores de sellos editoriales", "editores juveniles", "evaluadores”, booktubers? En el próximo apartado intentaremos ofrecer respuestas a estos interrogantes y abordaremos, con mayor detalle, las formas en las que la industria editorial y las tecnologías digitales se retroalimentan transformando el universo de la literatura juvenil.

\footnotetext{
${ }^{8}$ Andreo Rowling (España) tiene ı64.00o suscriptores, Javier Ruescas (España) tiene 289.ooo suscriptores Clau Read Books (México) tiene 455.Ooo suscriptores, por citar algunos ejemplos. Los tres han visitado Argentina en distintas ediciones de la FIL de CABA. Además de ser booktubers han devenido en escritores de literatura fantástica y juvenil.

9 Este término se utiliza para nombrar a aquellas personas que tienen una presencia importante en las redes sociales, que cuentan con muchos seguidores y que tienen gran prestigio entre ellos ya que valoran sus opiniones, fotografías, actividades, etc.

Io En el próximo apartado veremos cómo este momento se condice con una etapa de crecimiento de la industria editorial juvenil.
} 


\section{Estrategias del sector editorial: rupturas, continuidades y desafíos}

Para analizar el sector editorial juvenil en Argentina resulta necesario antes trazar un mapa de la situación actual del mercado del libro en el país. Entre los últimos años de la década de I990 y el 2000, tuvo lugar un proceso de concentración en el campo de la industria editorial argentina -así como en el resto de América Latina- a partir del cual los conglomerados de empresas que desembarcaron en el país llegaron a controlar la mayor parte del mercado. Como han señalado diversos estudios (Botto, 20o6; Szpilbarg y Saferstein, 2OI4a, 2OI4b; Muniz Jr y Szpilbarg, 2OI6; Szpilbarg, 2OI9) este proceso de concentración y globalización trasnacional siguió creciendo hasta la actualidad y estableció una disputa asimétrica por el control de los mercados entre los países.

Este proceso de "globalización editorial" (Sapiro, 2009) dio como resultado el predominio de dos grandes monopolios editoriales que se fueron apropiando también de los sellos más grandes de Argentina, a la vez que dominan los canales de venta y distribución. Por un lado, está el grupo PRH, del cual el multimedio alemán Bertelsmann adquirió las acciones en 2OI2, volviéndose la editorial más grande del mundo y uno de los holdings más importantes en medios y telecomunicaciones (Rama, 2003). Por otro, está el grupo multimedia y operador mundial en lengua castellana, Planeta, que también aglutina a más de cien empresas de áreas de negocios diferentes y a más de cincuenta editoriales.

Como explica Casanova (200I), la concentración generó que las editoriales se vean obligadas a responder a las exigencias de las empresas comunicacionales y de contenidos a las que pertenecen, por lo que se prioriza la rentabilidad a corto plazo, la aceleración en la rotación y el aumento constante de títulos. Esto implicó el avance del marketing y la publicidad en las editoriales para generar nuevas estrategias de venta, cruzadas con la utilización de las tecnologías de la información.

Por su parte, las publicaciones editoriales juveniles han crecido de forma exponencial en el mercado del libro argentino durante los últimos años. Según los informes de producción del libro argentino publicados por la Cámara Argentina del Libro (CAL), en el año 2OI4, la literatura infantil y juvenil (LIJ) ocupó el 2I\% del total de temáticas publicadas registradas en el sector editorial comercial, dentro de este porcentaje un IO\% corresponde exclusivamente a libros juveniles. Con tiradas de libros que llegan a los 50.00o ejemplares (como la saga de HP), en 2015 la LIJ llegó a ser la categoría más editada con el 23\% de la totalidad. En 2016 mantuvo ese porcentaje y liderazgo. Además, el informe arroja otro dato: la temática específicamente juvenil ocupa el $20 \%$ dentro de la categoría de la LIJ, es decir, que en dos años se duplicó ese tipo de ediciones. A su vez, los libros más vendidos durante ese año fueron dos títulos orientados al público juvenil: HP de J. K. Rowling y \#ChupaEIPerro, del youtuber Germán Garmendia. En 2OI\%, pese a que hubo una caída de ventas de la industria en el país y que el sector dejó de recibir compras del Estado, la LIJ continuó su primacía. Ese año la única distribuidora que en la Argentina mostró un crecimiento fue Riverside Agency, que subió un $29 \%$ sus ventas, porque 
Salamandra -a quien distribuye- publicó un nuevo libro vinculado al universo de HP que levantó la venta de toda la saga. Tanto en 2018 como en 2019 la LIJ siguió encabezando la lista de temáticas del mercado editorial argentino ${ }^{\text {I }}$.

Cabe señalar que, dentro de las publicaciones orientadas a este público, si bien se impone de manera contundente la presencia de los dos grupos antes mencionados, también se han destacado algunas editoriales medianas, como la española Salamandra (que de hecho en mayo de 2019 fue adquirida por PRH), o especializadas exclusivamente en literatura infantil y juvenil, -como lo son la Argentina V\&R y la colombiana Norma- que aplican estrategias similares de marketing y uso de las TIC.

Entre las tecnologías digitales y la industria editorial orientada al público juvenil se empezó a establecer un flujo de retroalimentación creciente. Este circuito se refleja en hechos de diversa naturaleza: jóvenes que empiezan a leer (y comprar los libros) por recomendaciones en redes de otros jóvenes, en general, y de BBB e influencers ${ }^{12}$, en particular; así como por el lanzamiento de versiones digitales de algunos libros, textos de la web que terminaron siendo editados en grandes tiradas de ejemplares, nuevos formatos de promoción del libro, como los booktrailers, entre otros fenómenos.

El desarrollo de las redes sociales, la generación de comunidades y las plataformas de lecturas permitieron un incremento exponencial en la difusión de libros juveniles para las editoriales. Quizás en menor medida, esto también se ha dado en otros sectores del mundo editorial. Como han señalado Szpilbarg y Saferstein (20I4a, 2OI4b), la industria editorial se encuentra sumergida en la actualidad en una etapa de cambios: por un lado, las nuevas tecnologías exigen a los editores otras competencias y acciones más allá de las tradicionales y por otro, los sellos editoriales despliegan estrategias de marketing que apuntan a detectar tendencias en su público de consumidores.

Como ya hemos mencionado, la proliferación de "comunidades virtuales" y "redes sociales" (Winocur, 20o6, 2009) permite a los jóvenes reelaborar sentidos de pertenencia e identificación ligados al deseo de traspasar las fronteras entre lo privado, individual y lo público, colectivo (Winocur, 20I3; Van Dijck, 20I6). Esto es así porque las TIC fomentan nuevas prácticas y modos de recepción y percepción (Yúdice, 2007). Junto a ello, se transforman también las pautas de producción y consumo. En ese sentido el papel de los BBB no se circunscribe sólo al interior de las comunidades, sino que tiene impactos en otros entramados sociales. Carla F., Community Manager de PRH hasta el año 20I8, explica esta relación entre nuevos mediadores literarios y la industria editorial juvenil a la luz de las transformaciones que tuvieron lugar en los últimos años:

\footnotetext{
${ }^{\text {II }}$ Datos recuperados de los informes de producción del libro argentino de los años 2OI4 al 2OI9 publicados por la Cámara Argentina del Libro. Obtenidos el to de mayo de 2020 desde http://www.camaradellibro.com.ar/index. $\mathrm{php/panorama-editorial/estadisticas.} \mathrm{Es} \mathrm{preciso} \mathrm{advertir} \mathrm{que} \mathrm{la} \mathrm{subdivisión} \mathrm{dentro} \mathrm{de} \mathrm{la} \mathrm{categoría} \mathrm{LIJ} \mathrm{con} \mathrm{el} \mathrm{des-}$ prendimiento de la categoría "juvenil" sólo aparece mencionada en los informes de producción del libro argentino del 2014 y del 2016.

I2 Como la escritora Magalí Tajes, quien utiliza su cuenta de Instagram para compartir lecturas de pasajes de sus libros Arde la vida y Caos, extensamente leídos entre jóvenes.
} 
Matías G.B., tenía el blog Cenizas de Papel y hoy colabora en el departamento de prensa, ayuda a pensar estrategias para acercarse a estos nuevos lectores. En Random se hizo un concurso de booktubers y bookstagrammers, se los convocó para trabajar en la editorial a través de la consultora. Estos chicos de i6 años al principio pedían a las editoriales libros, y con un paquete ya estaban felices. Después, ya con 20 años empezaron a estudiar marketing o edición. Ahí las editoriales vieron la oportunidad de contratar a gente que conocía su catálogo Young Adult, y que le agregaba valor. Por ejemplo, en 2015 hicimos en la Feria del Libro por primera vez un unboxing, en el canal "Me gusta Leer Argentina", en Youtube se puede ver. La actividad consiste en darle a un joven que se dedica a reseñar libros una caja con un libro y objetos vinculados a su historia (fragmento de entrevista con Carla, 2OI7).

Además del caso de Matías podemos mencionar otros ejemplos: Leonel Teti, que en 20II lanzó Sueños y Palabras, su blog dedicado a la difusión de la literatura juvenil, fue editor en V\&R, encargado del sector Young Adult, tanto en el país como en el exterior y desde fines de 20I7 fundó y es director editorial de Puck, el sello juvenil de Ediciones Urano, para América Latina y España. Su anterior puesto en VyR lo ocupa hoy Melisa Corbetto, editora y blogger de Lee. Sueña. Vuela. También Federico Valotta, fundador del blog de narrativa juvenil Atrapado en la lectura, pasó a ser asistente de marketing del sector juvenil en el grupo Planeta a principios de 2016.

En líneas generales podemos reconocer (al menos) tres niveles en los cuales los BBB se insertan y despliegan sus estrategias dentro de las editoriales: I) los que devenidos en influencers comparten, a través de sus cuentas, las últimas novedades literarias; 2) los que evalúan y corrigen manuscritos para las editoriales; y finalmente, 3) quienes dirigen sellos juveniles o tienen tareas como publisher $^{13}$. En términos de contratación, estos últimos tienen un vínculo salarial con las editoriales mientras que los freelances reciben una remuneración económica por su trabajo pero no forman parte de la editorial. Eliana W., editora y blogger lo comentaba en estos términos en el marco de una entrevista: "no soy de la editorial, osea todo el laburo de edición se hace freelance; y todo el mundo trabaja con un montón de editoriales". Efectivamente, este tipo de sistema de contratación es habitual para realizar la tarea de evaluadores, editores, traductores y diseñadores que se desempeñan en más de una editorial. En el caso de quienes "sólo comparten libros" el intercambio está dado por la posibilidad de asistir a presentaciones y Meet and greet con autores, por la oportunidad de dar charlas en ferias del libro y eventos literarios, así

I3 En inglés existen dos términos para referirse a la tarea del editor: el editor, propiamente dicho, que es quien realiza la tarea de edición en el manuscrito; y el publisher, aquella persona encargada de hacer los contactos con los autores, de gestionar sus contratos, de acompañarlos en sus giras publicitarias, entre otras demandas. Son una suerte de RRPP, en términos nativos. En el plano local, especialmente en editoriales pequeñas y medianas estas distintas actividades suelen solaparse. 
como por los propios libros. Está última forma de pago, a priori, simbólica también tiene un impacto en el bolsillo del BBB (o su familia) que se refleja, en términos nativos, en expresiones como "aliviana mucho la economía familiar", "mis papás están re contentos”I4. Estamos en presencia, entonces, de un sistema de intercambios en el que la recompensa salarial no es el único bien en juego y donde se disputan, a su vez, otro tipo de retribuciones.

En consecuencia, desde los sellos más pujantes en el sector juvenil, las acciones promocionales de libros se vinculan fuertemente con el mundo de los lectores adolescentes: invitan a los referentes, los apoyan materialmente, les dan lugar en sus sitios editoriales e incluso escuchan sus propuestas para futuras publicaciones.

Además, la experiencia de los unboxing que menciona la entrevistada refleja el uso de formatos audiovisuales para la promoción de los libros (que se da también en otras estrategias que no son exclusivas del sector juvenil, como los booktrailers). En este caso particular se apunta a llegar a los seguidores de los BBB invitados transmitiendo la experiencia en tiempo real en simultáneo en todos los sitios y dejándola luego a disposición para ser vista ${ }^{5}$. Si consideramos a la noción de paratexto en tanto elemento que ayuda al lector a introducirse en la lectura (Genette, I987) podríamos pensar el unboxing como una ampliación del concepto, con la incorporación de objetos y la difusión mediante una plataforma de Internet que permite la aparente conexión directa de la propuesta íntima con un lector.

Sin embargo, el rol de los BBB o jóvenes mediadores no se limita a la promoción lectora. Muchos BBB, ya consagrados como influencers en las redes, empezaron a publicar libros en las grandes editoriales. Podemos mencionar los casos de los españoles Andreo Rowling ${ }^{16}$ y Javier Ruescas, la primera con cuatro novelas ya publicadas y el segundo con más de veinte en apenas io años de trayectoria. También podemos mencionar a los mexicanos Claudia Ramírez y Alberto Villarreal, invitados al Encuentro Internacional de Booktubers de la FIL 20I9, espacio en el que publicitaron sus libros El príncipe del sol y Anoche en las trincheras, respectivamente, ambos editados por Planeta en 20I8. En el plano local, podemos destacar el caso de Érase una vez, una antología de relatos clásicos reversionados por miembros de la comunidad BBB, publicada por el mismo sello en

\footnotetext{
${ }^{\text {I4 }} \mathrm{Al}$ mes de noviembre de 2OI9, un libro de Planeta o PRH, de más de 3oo páginas, en una edición estándar oscila entre los i2 y los i6 dólares; dependiendo, en gran medida, de la procedencia del autor (los BBB suelen leer autores extranjeros, lo que en general aumenta el valor del libro). El salario promedio de un joven de entre 20 y 34 años en el tercer trimestre de $20 \mathrm{I}_{9}$ en Argentina es de 7 I5 dólares. Si consideramos un grupo familiar de 4 personas (teniendo en cuenta que muchos $\mathrm{BBB}$ aun viven con sus padres) este número asciende a alrededor de 3.ooo. En el marco de esta economía, la compra de (al menos) 4 libros, que es el mínimo que suelen leer por mes estos jóvenes, implica unos 60 dólares mensuales unicamente en libros. La compra de ebooks puede ser más económica (en algunos casos a mitad de precio), pero no permite la misma promoción del libro "en papel” a través de las redes sociales por lo que no suele ser la primera opción entre los BBB. En resumidas cuentas, hablamos de valores económicos significativos para la economía doméstica.

I5 En el video referido por la entrevistada se puede ver al booktuber Federico Valotta sacar de la caja hojas, lapiceras, macilla, un lápiz labial y telas de colores, todos objetos relacionados con la historia del libro promocionado, Te daría el mundo. Obtenido el Io de mayo de 2020 desde https://www.youtube.com/watch? $=$ M 43 KmtuewVo

${ }^{\text {I6 }}$ Bautizada como Andrea Izquierdo Fernández, toma el apellido Rowling de la autora de HP.
} 
2OI7, o Todxs somos normales escrito por dos jóvenes bloggers, y publicado por Ediciones Destino en 20I8. Recientemente, en 20I9, varios miembros de la comunidad BBB (algunos de ellos devenidos en editores, como Melisa Corbetto y Leonel Teti), autoras ya consagradas en el género fantástico/juvenil (como Tiffany Calligaris, escritora argentina que actualmente reside en Canadá) así como escritoras y editoras con una trayectoria más extensa en este ámbito (como Cristina Alemany, coordinadora del área Juvenil de Fundación El Libro o Fabiana Scherer, periodista que desde hace años escribe sobre literatura juvenil en distintos medios masivos de comunicación) publicaron, con la editorial Catapulta, Esos raros relatos nuevos, una compilación de cuentos que buscan interpelar al público juvenil.

Por su parte, Wattpad también ha sido percibida como un semillero de escritores por el campo editorial argentino. En efecto, los textos que lograron muchas vistas en la red fueron editadas en enormes tiradas de libros por grandes sellos. El primer caso fue la novela After, de Anna Todd, que había logrado millones de vistas en la plataforma, y fue publicada por Planeta en 20I5, transformándose en un fenómeno de ventas en el mundo. Hay otros casos similares, en la actualidad, en Argentina podemos mencionar a Jazmín Riera, que, como dice su biografía en la web de Planeta, "a los diecisiete subió a la plataforma Wattpad la novela Las reglas del boxeador y rápidamente se convirtió en una de las ficciones más leídas" "'7. Como explica Van Dijck, existe un sistema de recomendaciones, jerarquía y popularidad que imponen las plataformas virtuales y "un usuario [que] se convierte al mismo tiempo en proveedor de recursos, productor y consumidor" (2OI6: 274). Ambos rasgos, condensados en la categoría de prosumidor, fueron registrados por el mercado editorial. En consecuencia, las editoriales han tenido que adaptarse a estos nuevos formatos y estos nuevos lectores, activos y participativos, que interactúan con los autores, leen, comentan y publican sus producciones literarias. No hay una unidireccionalidad absoluta de la propuesta editorial a los jóvenes, existe una retroalimentación, e, incluso, son ellos mismos quienes marcan el camino. Como señala Fernanda S., encargada del sector de ventas de Random House hasta el año 2OI8:

Los juegos del hambre fue como HP. Se agotaba y la editorial que lo distribuía (RBA) llegaba más tarde que lo que el fenómeno había picado. Ahí nos dimos cuenta que los chicos estaban leyendo mucho más rápido que lo que se editaba. La editorial se dio cuenta que estaba pasando algo que no estaba viendo y corrió detrás (fragmento de entrevista con Fernanda, 20I9).

De este último testimonio, así como de lo descripto hasta aquí, se desprenden varias cuestiones. En primer lugar, el borramiento creciente de los límites entre el autor

I7 Obtenido el I2 de noviembre de 2019 desde https://www.planetadelibros.com.ar/autor/jazmin-rie$\mathrm{ra} /$ oooO 46289 
y el lector. En segundo lugar, que el uso de los formatos web se han vuelto una cuestión central para la difusión y construcción de la imagen de la editorial (Szpilbarg, 20I9) y obligan al editor a llevar adelante otras tareas que no tienen que ver con la selección de contenidos, sino con la interacción con lectores y autores desde un rol activo en las redes. Finalmente, en el campo de la lectura y la escritura, se revela que las culturas jóvenes -segmento que ha crecido en los últimos años en todos los mercados incluyendo el editorial- se muestran cada vez más autónomas con sus consumos culturales propios.

En consecuencia, asistimos a un proceso de transformaciones dentro de la industria editorial, en el que se despliegan nuevas formas de editar pero también nuevas formas de mirar el negocio. En una industria de bienes culturales, a la que se le adosan toda una serie de representaciones y prácticas, y en la que prima una valoración construida colectivamente en torno libro al como objeto; se cruzan discursos residuales y también emergentes. En un mundo donde la lógica cultural, simbólica, intelectual parecía primar llegan editores, mucho menos culpógenos en relación a la dimensión económica del libro, que le aportan un nuevo dinamismo a esta industria con prácticas que reditúan económicamente a editoriales y aportan una nueva visión del negocio. Al mismo tiempo, estos mismos jóvenes BBB continúan estudiando la carrera de Edición en la Universidad para ocupar cargos como editores dentro de esta particular industria, evidenciando el peso simbólico que esta figura todavía tiene en sus discursos e imaginarios. De este modo, notamos que hay una cierta reproducción de ese mundo de la edición que expresa esas tensiones no resueltas entre lógicas comerciales y culturales intrínsecas en estas empresas de producción cultural.

\section{Conclusiones}

En el presente artículo nos propusimos cruzar las prácticas de lectura y las prácticas en torno a la apropiación de nuevas tecnologías en relación a la edición de libros de literatura juvenil de circulación masiva, segmento que creció a contramano de la caída generalizada de la industria editorial. Corrernos de la pregunta “¿por qué los jóvenes no leen?” e interrogarnos, en cambio, “¿cómo es que los jóvenes leen y qué tipo de vínculo entablan con lo literario?” nos coloca, en primer lugar, en la necesidad de describir y comprender qué estrategias se despliegan desde el mundo editorial para promover estas experiencias lectoras. En consecuencia, en este trabajo nos centramos en un universo de nuevos editores o nuevos agentes vinculados al mundo de la edición (muchos de ellos parte de lo que se autodenomina comunidad BBB), espacio en el que conviven agentes, prácticas y representaciones que revelen una transformación a partir de la reconfiguración del espacio editorial (Saferstein y Szpilbarg, 2OI4a, Szpilbarg, 20I9). Notamos que se perciben cambios en el mercado del libro, en el ecosistema de las editoriales: fusiones, concentraciones, nuevos emprendimientos, una necesaria intervención de las nuevas tecnologías, nuevas prácticas y tipos de expertise, nuevos modos de editar, nuevas 
formas de buscar la creatividad en torno a la búsqueda de temas y autores potenciales, que le aportan un dinamismo especial a esta industria. Como planteamos, los alcances de estas transformaciones todavía están por verse. Y esto reviste una complejidad particular ya que, en este escenario, el mercado no tiene un dominio absoluto sobre el público lector, sino que muchas veces se ve forzado a seguir sus tiempos, sus formas y sus demandas. Las nuevas tecnologías imponen cambios y aún estamos en una etapa de transición e incertidumbre. Los lectores y consumidores, devenidos en prosumidores, redefinen los vínculos entre el universo digital y el mercado del libro.

Como ya mencionamos, el desarrollo de investigaciones situadas, con un enfoque transdisciplinar que involucre diversas áreas del conocimiento, se impone como indispensable para poder describir y analizar las transformaciones en la circulación, recepción y creación de la literatura entre jóvenes en contextos tecnologizados y las exigencias del mercado editorial, que puja por conquistarlos. ¿Cuánto hay de nuevo y cuánto de permanencia en torno a estos fenómenos? Esta es una pregunta que nos invita a una reflexión continua y situada en experiencias empíricas para seguir analizando este particular universo. 


\section{Referencias bibliográficas}

- Albarello, F.; Arri, F.; García Luna, A. (2018). Booktubers: nuevos jugadores en el mercado del libro. Intercambios, La letra del encuentro, 4(2), I9-25. Obtenido el 4 de mayo de 2020 desde https://issuu.com/posgradounq/docs/intercambios.laletradelencuentro.iv_2_

- Baricco, A. (2019) The Game. Barcelona: Anagrama.

- Botto, M. (2006). 1990-2000. La concentración y la polarización de la industria editorial. En De Diego, J. L. (dir.). Editores y políticas editoriales en Argentina, I880- 2000 (pp. 2I9270). Buenos Aires: Fondo de Cultura Económica.

- Casanova, P. (20OI). La república mundial de las letras. Barcelona: Anagrama.

- Cassany, D. (2OII). En_linea. Leery escribir en la red. Barcelona. Anagrama.

- Corea, C. y Lewkociz, I. (2004). Pedagogía del aburrido. Escuelas destituidas, familias perplejas. Buenos Aires: Paidós.

- Castells, M. (2OOI). Internet y la sociedad red. Madrid: Alianza editorial.

- Cuesta, C. y Sawaya, S. (comp.) (20I6). Lectura y escritura como prácticas culturales. La investigación y sus contribuciones para la formación docente. La Plata: Edulp.

- De la Torre-Espinosa, M. (2O2O). El fenómeno Booktube, entre el fandom y la crítica literaria. Álabe, 2I, I-IO.

-Dubet, F. (2002). Le declin del'institution. Paris: Seuil.

- Dussel, I. (2OI2). Más allá del mito de los “nativos digitales”. Jóvenes, escuela y saberes en la cultura digital. En Southwell, M. (comp.). Entre generaciones. Exploraciones sobre educación, cultura e instituciones (pp. I83-2I3). Rosario: FLACSO/Homo Sapiens.

-Escandell, D. (20I6). La literatura digital. Revista Peonza, п18, 5-16.

- Ferreiro, E. (2OII). Alfabetización digital, ¿de qué estamos hablando?. Educação e Pesqui$s a, 37(2), 423-43^{8}$.

- García Canclini, N. (2015) Leer en papel y en pantallas: el giro antropológico. García Canclini, N. (comp.). Hacia una antropología de los lectores (pp. I-35). México: Ediciones culturales Paidós.

- Genette, G. (1987). Seuils. Paris: Seuil. 
- Gaarder, J. (2006). ¿Libros para un mundo sin lectores? Educación y Biblioteca, I53. Obtenido el 4 de mayo de 2020 desde: https://gredos.usal.es/bitstream/handle/ıo366/ır9248/ EBı8_Ni53_Pi7-2o.pdf;jsessionid=AiCF7ıEDD8A3r864E9E573F937 $\mathrm{E}_{3} \mathrm{~B}_{4} 67$ ? sequen $\mathrm{ce}=\mathrm{I}$

- Groys, B. (20I4). Volverse público. Buenos Aires: Caja Negra.

- Jenkins, H. Convergence Culture. La cultura de la convergencia de los medios de comunicación. Barcelona: Paidós.

- Kessler, G. (2002). La experiencia escolar fragmentada. Estudiantes y docentes en la escuela media en Buenos Aires. Buenos Aires: IIPE - UNESCO.

- Kessler, G. (2004). Sociología del delito amateur. Buenos Aires: Paidós.

- Kozac, C. (20r8). Comunidades experimentales y literatura digital en Latinoamérica. Virtualis, 9 (I7), 9-35.

- Lahire, B. (2004). Sociología de la lectura. Barcelona: Gedisa.

- Lluch, G. (2OI4). Jóvenes y adolescentes hablan de lectura en la red. Ocnos, II, 7-2O.

- Melo Moreira, R. P. (20I8). Booktubers e mercado editorial: parcerias como estratégia de colonização dos espaços de referência. Actas de Intercom - 41 Congresso Brasileiro de Cièncias da Comunicação de lá UNIVILLE. Congreso llevado a cabo en Joinville - S.C. Obtenido el Io de mayo de 2020 desde http://portalintercom.org.br/anais/nacional2or8/resumos/ Ri3-2IO7-I.pdf

- Merino, P. y Polanco, J. L. (2015). Formar lectores en la era digital. Madrid: Ediciones de la Torre.

- Muniz Jr, J. D. S. y Szpilbarg, D. (2OI6). Edição e tradução, entre a cultura e a política: Argentina e Brasil na Feira do Livro de Frankfurt. Sociedade e Estado, 3I(3), 67 I-692.

- Pacheco Alonso, B. (2OI4). Booktube. Una propuesta para el fomento a la lectura. UNAM. Obtenido el 4 de mayo de 2020 de: https://issuu.com/gustavopacheco $4 /$ docs/booktubers_linea_

- Papalini, V. (2OI2a). Aproximaciones a los modos de leer: sobre la lectura como experiencia, como práctica y como herramienta. Actas de las VII Jornadas de Sociología de la UNLP. Congreso llevado a cabo en Ensenada. Obtenido el ro de mayo de 2020 desde http://jornadassociologia.fahce.unlp.edu.ar/vii-jornadas-2OI2/actas/Papalini.pdf/view 
- Papalini, V. (2OI2b). Las lecciones de los lectores. A propósito de la recepción literaria. Alabe, 6, I-2I.

- Papalini, V. (20I5). Sobre la lectura como experiencia, como práctica y como herramienta. En Muse, C. (ed). Lectura y escritura: continuidades, rupturas y reconstrucciones (pp. 4I- $\left.{ }^{\mathrm{I}} \mathrm{I}\right)$. Córdoba: Universidad Nacional de Córdoba.

- Papalini, V.(2020). La escritura escuchada. Circulación literaria y recreación de comunidad. En Boix, O y Iuliano, R (comp.). La cultura como dimensión transversal de lo social: objetos, conceptos y debates actuales acerca de los mundos del arte contemporáneos. FaHCEUNLP, CLACSO (En prensa).

- Pates, G. (2OI5). ¿Los jóvenes no leen? Experiencias de lecturas en booktubers. Revista Letras,I, I25-I3I.

- Peirano, M. (2004). A favor de La etnografía. En Grimson, A., Lins Ribeiro, G. y Semán, P. (comp.), La antropología brasilera contemporánea. Contribuciones para un diálogo latinoamericano. Buenos Aires: Prometeo.

- Pini, M.; Amaré, M.; Cerdeiro, C. y Terzian, C. (20r6). Consumos digitales de los jóvenes: ¿puentes o muros para la enseñanza escolar? Revista Propuesta Educativa, 46(2), 84-92.

- Piovani, J. I. (20I8). La entrevista en profundidad. En Marradi, A.; Archenti, N. y Piovani, J. I. (comp.). Manual de Metodología de las Ciencias Sociales (pp. 265-278). Buenos Aires: Siglo XXI.

- Pindado, J. (2004). El desencuentro entre los adolescentes y la lectura. Comunicar, 23, I67-I72.

- Rama, C. (2003). Economía de las industrias culturales en la globalización digital. Buenos Aires: Eudeba.

- Rovira Collado, J. (2OI7). Booktrailer y Booktuber como herramienta LIJ 2.0 para el desarrollo del hábito lector. Investigaciones sobre lectura, 7, 55-72.

- Sadin, É. (20I8). La silicolonización del mundo. Buenos Aires: Caja Negra.

- Saez, V. (20I9). De las pantallas al papel. Nuevos acercamientos de las jóvenes a la literatura. El Toldo de Astier, Io (18), 42-51.

- Saferstein, E. y Szpilbarg, D. (20I4a). Experiencias de trabajo en el capitalismo informacional. El caso de la industria editorial Argentina. Trabajo y sociedad, 22, 257-27I. 
- Saferstein, E. y Szpilbarg, D. (2OI4b). La industria editorial argentina, I99O-2OIO: entre la concentración económica y la bibliodiversidad. Alternativas, 3, I-2I.

- Sapiro, G. (2009). Les contradictions de la globalisation éditoriale. París: Nouveau Monde.

- Scolari, C. (20I3). Narrativas transmedia. Barcelona: Deusto.

- Seman, P. (2015). Música, juventud, hegemonía: crítica de una recurrencia. Apuntes de investigación del CECYP, 17 (25), II9-I46.

- Sibilia, P. (2008). La intimidad como espectáculo. Buenos Aires: Fondo de Cultura Económica.

- Szpilbarg, D. (20I9). Cartografía argentina de la edición mundializada. Modos de hacery pensar el libro en en siglo XXI. Buenos Aires: Tren en Movimiento.

- Toffler, A. (1980). La tercera ola (The third wave). México: Edivisión.

- Torti Frugone, Y. y Schandor, A. M. (20I3). El reino más grande del mundo: la existencia del fandom como fenómeno cultural. Actas de VII Jornadas de Jóvenes Investigadores del Instituto de Investigaciones Gino Germani de la UBA. Jornadas llevadas a cabo en Buenos Aires. Obtenido el Io de mayo de 2020 desde https://www.aacademica.org/ooo-o76/98.pdf

-Valles, M. (I997). Técnicas de conversación, narración (I): las entrevistas en profundidad. En Valles, M. (comp.). Técnicas cualitativas de investigación social. Reflexión metodológica y práctica profesional. (I77-243). Madrid: Síntesis.

-Van Dijck, J. (20I6). La cultura de la conectividad. Buenos Aires: Siglo XXI.

- Visacovsky, S. (2008). Estudios sobre “clase media” en la antropología social: una agenda para la Argentina. Avá, Revista de Antropología, ı3, 9-37.

- Winocur, R. (2006). Internet en la vida cotidiana de los jóvenes. Revista mexicana de sociología, $68(3), 55^{\mathrm{I}-} 5^{80}$.

- Winocur, R. (2009). Robinson Crusoe ya tiene celular: la conexión como espacio de control de la incertidumbre. México: Siglo XXI.

- Winocur, R. (20I3). La intimidad de los jóvenes en las redes sociales. Telos. Fundación Telefónica, 9. Obtenido el 4 de mayo de 2020 desde https://telos.fundaciontelefonica.com/ archivo/numeroogi/la-intimidad-de-los-jovenes-en-las-redes-sociales/

- Yúdice, G. (2007). Nuevas tecnologías, música y experiencia. Barcelona: Gedisa. 